\section{Nouvelles de l'industrie Neues aus der Industrie}

\section{Efalizumab - Le contrôle à long terme bien toléré}

\author{
Meilleure qualité de vie \\ avec le psoriasis: des agents \\ biologiques pour une prise en \\ charge optimale des patients
}

Un symposium qui s'est tenu dans le cadre de l'assemblée annuelle de la SSDV à Genève («How to manage the new treatment in psoriasis», lunch symposium à l'occasion de la 88e Réunion annuelle de la SSDV à Genève, au Centre médical universitaire, soutenu par Serono Pharma Suisse) est arrivé à la conclusion suivante: «L'anticorps monoclonal éfalizumab (Raptiva ${ }^{\circledR}$ ) est une option thérapeutique à long terme et importante pour les patients atteints d'un psoriasis en plaques qui ne répondent pas de manière satisfaisante à un traitement systémique conventionnel ou qui le supportent mal.» De fait, l'éfalizumab occupe une place particulière dans le traitement des patients atteints de psoriasis, car son profil de sécurité est exceptionnel, explique le Dr Nicole Selenko-Gebauer, spécialiste d'immunodermatologie de l'Université de médecine de Vienne. Les aspects à prendre en considération lors de la prise en charge en cabinet des patients ont été discutés en détail par les experts présents lors du symposium.

En Suisse, la prévalence du psoriasis au sein de la population se situe autour de $2 \%$. Jusqu'il y a peu, le psoriasis était complètement sous-estimé, aussi bien par le public que même par les dermatologues. Un grand nombre de dermatologues continuent à considérer le psoriasis comme un "problème cosmétique». Les personnes atteintes de psoriasis sont pourtant fortement gênées dans leur vie quotidienne et connaissent de grandes souffrances.

La plupart des patients souffrent de la forme légère, qui peut être traitée de manière satisfaisante au moyen de médicaments topiques. Dans les cas modé- rés à sévères, la luminothérapie et le traitement systémique [1] se sont imposés, et sont actuellement devenus les traitements standard. Ces méthodes sont toutefois limitées par l'existence de contre-indications strictes. Des effets secondaires spécifiques sont en outre possibles. De plus, ces traitements ne satisfont pas toujours les besoins des patients. L'éfalizumab (Raptiva) est le premier agent biologique à avoir été admis en Suisse, en 2004, et il est pris en charge par les caisses-maladie depuis janvier 2005.

Le Prof. Jean-Hilaire Saurat, chef du service de dermatologie et de vénéréologie de l'Hôpital universitaire de Genève et président du symposium, présente l'importante évolution du traitement du psoriasis ces dernières années, marquée principalement par l'introduction des agents biologiques, qui présentent une efficacité et une tolérance élevées chez les patients atteints de psoriasis modéré à sévère. L'objectif du symposium intitulé «How to manage the new treatment in psoriasis» était d'échanger les expériences pratiques acquises lors de l'utilisation de cette option thérapeutique contre le psoriasis.

Le Prof. Mona Stähle du Karolinska Institut, Stockholm (Suède), souligne le fait que les patients qui souffrent longtemps d'un psoriasis sévère présentent une morbidité et une mortalité cardiovasculaire plus élevées [2, 3]. Les facteurs de risque comme un profil lipidique anormal, une hypertension artérielle, une obésité et un diabète sont étroitement associés à un psoriasis sévère suggérant que le psoriasis est en fait plus q'une maladie de la peau. Il convient de choisir le traitement en prenant en considération ce risque cardiovasculaire potentiellement élevé chez ces patients. Les inhibiteurs du TNF- $\alpha$ sont par exemple contre-indiqués en cas d'insuffisance cardiaque. Les rétinoïdes ainsi que la ciclosporine peuvent provoquer une augmentation des taux sanguins de lipides.

\section{Qu'est-ce qui rend l'agent biologique éfalizumab si spécial?}

L'éfalizumab, anticorps monoclonal humanisé, module les cellules T sans les détruire, l’organisme reste capable de se défendre contre les infections. D'autres agents biologiques peuvent affaiblir le système immunitaire, les patients ont ainsi un risque nettement plus élevé d'infection. L'éfalizumab bloque les trois principales étapes du psoriasis en plaques: au niveau des ganglions lymphatiques, il empêche tout d'abord l'activation de cellules $T$ naïves par des cellules présentatrices d'antigènes; ensuite, la liaison des cellules $\mathrm{T}$ aux cellules endothéliales dans les vaisseaux est inhibée, et leur migration dans le derme est bloquée; finalement, l'éfalizumab inhibe la réactivation des cellules $\mathrm{T}$ de mémoire dans la peau par des cellules présentatrices d'antigènes, et empêche ainsi la libération de cytokines. L'éfalizumab est donc le seul modulateur réversible des cellules $T$ qui intervient spécifiquement dans le processus de la maladie. En outre, l'éfalizumab est simple à utiliser. Les patients, après un apprentissage rapide, procèdent eux-mêmes à l'injection sous-cutanée une fois par semaine.

Important: faire la distinction entre les répondeurs et les non-répondeurs

L'efficacité et la tolérance de l'éfalizumab ont été évaluées au cours de plusieurs études de phase III, contrôlées par placébo, chez des patients souffrant de psoriasis modéré à sévère. Jusqu'ici, plus de 3500 patients ont été traités dans le cadre d'études cliniques durant 1-3 années. Les données concernant l'efficacité, recueillies lors d'études contrôlées par placébo [4-7], montrent qu'après 12 semaines de traitement, le PASI (Psoriasis Area and Severity Index) est de 75 (réduction de 75\%) chez un pourcentage pouvant aller jusqu'à 39\% des patients et il est de 50 (réduction de $50 \%$ ) chez un pourcentage pouvant atteindre $61 \%$ des patients. En outre, 30\% des patients qui n'avaient pas ou plus répondu à un traitement systémique standard ou chez lesquels celui-ci était contre-indiqué ont présenté un PASI 75 après 12 semaines de traitement [6]. Les répondeurs ont présenté un effet net au cours des 2 premières semaines de traitement, ce qui laisse supposer que le début de l'action de l'éfalizumab est rapide.

Le prolongement du traitement audelà du traitement initial augmente le 


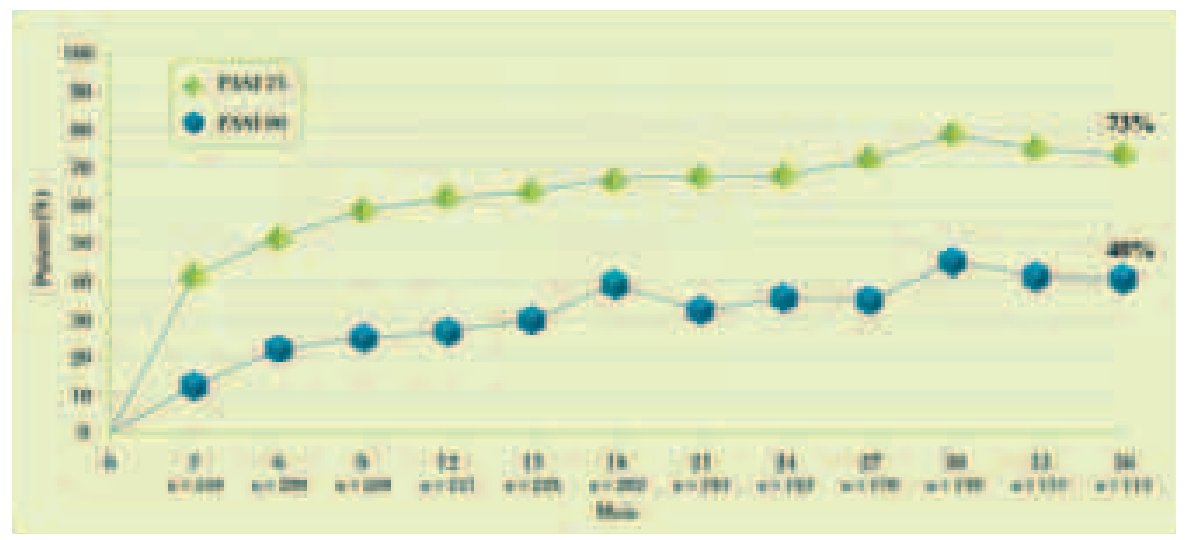

Fig. 1. Taux de réponse PASI 75 et PASI 90 durant un traitement à long terme de 3 ans dans une analyse «as treated» [9].

pourcentage de répondeurs: après 24 semaines de traitement, $44 \%$ des patients présentaient déjà un PASI 75 et $67 \%$ un PASI 50 [8].

Lors d'un traitement à long terme, sur 36 mois, un total de $73 \%$ des patients ont présenté une amélioration de $75 \%$ (PASI 75) de l'état de la peau et 40\% une amélioration de 90\% (PASI 90) dans une analyse «as treated» (fig. 1).

Selon le Dr Selenko-Gebauer, il est important de savoir que d'après les données disponibles actuellement, l'éfalizumab n'a pas d'effet sur une éventuelle arthrite psoriasique concomitante. Les patients atteints de ce problème articulaire nécessitent donc soit l'association d'un AINS à l'éfalizumab, soit l'utilisation d'un autre agent biologique efficace également contre ces troubles. La décision quant à l'éventuelle poursuite de l'éfalizumab est prise au cours des 12 premières semaines de traitement. Lorsque l'état de la peau s'améliore durant cette période, l'efficacité du traitement pourra même augmenter au cours du temps. Selon le Dr Selenko-Gebauer, un traitement qui reste inefficace après 12 semaines laisse supposer que le patient ne répond pas au médicament et justifie l'interruption du traitement. A noter qu'environ $6-8 \%$ des patients atteints de psoriasis et traités par éfalizumab sont des «non-répondeurs inflammatoires». Chez ces patients, les symptômes cutanés inflammatoires empirent au lieu de s'améliorer sous traitement, explique la dermatologue. Si l'on constate une telle évolution paradoxale, le médicament doit être arrêté après 8 semaines au plus tard.

\section{Les tentatives d'interruption mettent en jeu la réponse à long terme}

Une poussée de la maladie au cours d'un traitement avec l'éfalizumab après une première réponse ne nécessite pas d'interrompre immédiatement le médicament, souligne le Dr Selenko-Gebauer. Ces patients devraient plutôt bénéficier de traitements complémentaires pendant un certain temps. Une option consiste à administrer 15-20 mg de méthotrexate par semaine pendant 4-6 semaines ou à prescrire un traitement intense par voie locale en ambulatoire. Le Dr Selenko-Gebauer met en garde contre le risque de perdre la réponse à long terme avec l'éfalizumab si l'on essaie de diminuer la dose recommandée (1 $\mathrm{mg}$ / $\mathrm{kg}$ /semaine par voie sous-cutanée) ou d'allonger les intervalles entre les doses (hebdomadaires). En effet, dans ces situations, il faut s'attendre à une aggravation de la symptomatologie cutanée.

Léfalizumab convient pour un traitement durable, c'est-à-dire que le patient n'arrête plus le médicament. Il ne doit pas être utilisé durant la grossesse. Les vaccinations vivantes sont à éviter.

\section{Bon profil de sécurité et de tolérance}

Parmi les effets indésirables du traitement, on trouve les symptômes pseudo-grippaux aigus légers à modérés, qui sont toutefois considérés comme «supportables» par la plupart des patients, et qui peuvent être traités facilement par le paracétamol. Ces symptômes s'atténuent au cours du traitement. Les expériences acquises à ce jour ont montré que des papules, similaires à ceux du psoriasis guttata, sont susceptibles d'apparaître lors du traitement avec l'éfalizumab, particulièrement chez les répondeurs: ces symptômes apparaissent généralement dans la région axillaire et inguinale, 4-8 semaines après le début du traitement; ils disparaissent grâce à l'emploi de traitements topiques, sans imposer l'interruption du traitement par éfalizumab. Lors d'une utilisation à long terme, aucun nouvel effet secondaire n'apparaît. Aucun risque de tumeur n'a été constaté au cours d'une durée d'observation de 3 ans sur un collectif de 150 patients. Des infections sévères - comme sous tous les agents biologiques - sont apparues chez $0,4 \%$ des patients - un taux tout à fait «raisonnable».

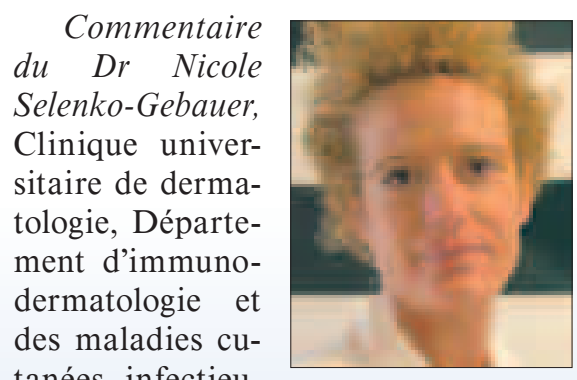
tanées infectieuses, Service externe pour le psoriasis et les dermatoses auto-immunes, Université de médecine de Vienne:

Un agent biologique est une protéine qui intervient de manière ciblée dans les processus clés du psoriasis. C'est pourquoi l'action présente une spécificité encore jamais atteinte à ce jour et une tolérance nettement supérieure aux autres traitements. Le traitement systémique du psoriasis était dominé jusqu'ici par des médicaments qui avaient des effets secondaires sévères. Aucun de ces médicaments ne pouvait être utilisé à long terme sans problème. C'est pourquoi tous les traitements étaient pulsés. En effet, il n'était pas possible d'imposer un traitement durable aux patients. Depuis l'introduction en dermatologie des agents biologiques, nous disposons d'un traitement à long terme bien toléré.

Un principe de base de l'utilisation d'agents biologiques est la reconnaissance des effets secondaires à évolution parfois très subtile - il appartient au spécialiste de les suivre, car il s'agit d'un 
travail difficile, demandant un grand sens des responsabilités.

Le consensus autrichien préconise un traitement par les agents biologiques chez les patients atteints de psoriasis modéré à sévère - par définition, des patients avec un PASI $>12$ ou une fraction de la surface corporelle atteinte supérieure à $10 \%$. À mon avis, ce ne sont pas les seuls cas de psoriasis susceptibles d'être considérés comme "sévères». Le patient atteint de psoriasis pustuleux palmoplantaire par exemple fait lui aussi partie de cette catégorie, même s'il ne remplit pas les critères cités. Les patients souffrent énormément. Certains d'entre eux ne peuvent plus marcher ou travailler. C'est pourquoi je considère que ces patients sont également des candidats pour les agents biologiques. Il existe des exemples de patients atteints de psoriasis palmoplantaire traités avec succès [10].

En ce qui concerne la question des coûts des agents biologiques: il ne s'agit certainement pas d'administrer un agent biologique dans les cas légers, pour lesquelles un traitement local suffit. Si, en administrant un agent biologique, je parviens à améliorer considérablement la qualité de vie d'un patient traité depuis 15 ans par PUVA et chez lequel les médicaments habituels provoquent des nausées sévères, alors l'utilisation de ces agents est sans doute justifiée.

Pour nous, l'éfalizumab occupe une place particulière, car son profil de sécurité est exceptionnel. En fait, il n'existe à ce jour aucun produit comparable. Dans notre clinique, nous traitons actuellement près de 200 patients par éfalizumab. Nous n'avons connu aucun problème d'infection opportuniste ou de néoplasie, et nous n'avons observé aucune perte d'efficacité chez les patients. Nous administrons également éfalizumab à des patients souffrant de psoriasis et présentant soit une tuberculose latente, soit une pathologie cardiaque ou hépatique associée. En principe, nous l'utilisons en tant qu'agent biologique de première intention. Les candidats typiques pour l'éfalizumab sont les patients atteints d'un psoriasis tout à fait modéré à sévère, sans problème articulaire associé.
Rapport: Dr Ellen Heitlinger,

$\mathrm{H}+\mathrm{O}$ communications ag, Baar

Avec le soutien de

Serono Pharma Suisse

Steinhauserstrasse 74 , case postale

CH-6301 Zoug

info@psoriasisweb.ch

www.raptiva.ch

\section{Bibliographie}

1 Nickoloff BJ, et al: Recent insights into the immunopathogenesis of psoriasis provide new therapeutic opportunities. J Clin Invest 2004;113:1664-1675.

2 Malbris L, et al: Psoriasis is associated with lipid abnormalities at the onset of skin disease. J Am Acad Dermatol 2006; 54:614-621.

3 Henseler T, et al: Disease concomitance in psoriasis. J Am Acad Dermatol 1995;32: 982-986.

4 Lebwohl MG, et al: A novel targeted T-cell modulator, efalizumab, for plaque psoriasis. N Engl J Med 2003;349:2004-2013.

5 Gordon KB, et al: Efalizumab for patients with moderate to severe plaque psoriasis: a randomized, controlled trial. JAMA 2003;290:3073-3080.

6 Sterry W, et al: Clinical Experience Acquired with Raptiva (CLEAR) trial in patients with moderate-to-severe plaque psoriasis: results from extended treatment in an international, phase III, placebo-controlled trial. JDDG, sous presse.

7 Leonardi CL, et al: Extended efalizumab therapy improves chronic plaque psoriasis: results from a randomized phase III trial. J Am Acad Dermatol 2005;52:425433.

8 Menter A, et al: Efficacy and safety observed during 24 weeks of efalizumab therapy in patients with moderate to severe plaque psoriasis. Arch Dermatol 2005; 141:31-38

9 Poulin Y, et al: A favourable benefit/risk ratio with efalizumab: a review of the clinical evidence. J Cutan Med Surg 2005; 1 : 10-17.

10 Fretzin SY, et al: Efficacy and safety of efalizumab for patients with palmoplantar pustulosis (poster). Meet Am Acad Dermatol, New Orleans, février 2005.

\section{Efalizumab - die gut verträgliche Langzeitkontrolle}

\author{
Mehr Lebensqualität \\ bei Psoriasis: Biologika \\ für ein optimales \\ Patientenmanagement
}

Für Patienten mit einer Plaque-Psoriasis, die auf eine konventionelle systemische Therapie nicht befriedigend ansprechen oder sie schlecht vertragen, ist der monoklonale Antikörper Efalizumab (Raptiva ${ }^{\circledR}$ ) eine wichtige langfristige Therapieoption, so das Fazit eines Symposiums während der Jahresversammlung der SGDV in Genf («How to manage the new treatment in psoriasis», Lunch-Symposium anlässlich der 88. Jahresversammlung der SGDV in Genf am Centre médical universitaire, unterstützt von Serono Pharma Schweiz). Denn Efalizumab habe einen besonderen Stellenwert in der Behandlung der Psoriasispatienten, da das Sicherheitsprofil aussergewöhnlich sei, erläutert Dr. Nicole Selenko-Gebauer, Fachärztin für Immundermatologie an der Medizinischen Universität Wien. Was es beim Patientenmanagement in der Praxis zu beachten gilt, wurde auf dem Symposium unter den anwesenden Experten eingehend diskutiert.

In der Schweiz liegt die Psoriasisprävalenz der Bevölkerung etwa bei $2 \%$. Die Psoriasis ist in der Öffentlichkeit, aber auch bei den Hautärzten selbst, noch bis vor kurzem völlig unterschätzt worden. Viele Dermatologen halten Schuppenflechte immer noch für ein «kosmetisches Problem». Psoriatiker sind in ihrem Leben stark eingeschränkt und stehen unter einem enormen Leidensdruck.

Die Mehrzahl der Patienten ist von einer leichten Form betroffen, die mit topischen Medikamenten zufrieden stellend behandelt werden kann. Die mittelschweren bis schweren Fälle werden standardmässig mit einer Lichtund einer systemischen Therapie behandelt [1], die ihren festen Platz haben. Allerdings existieren dafür einschneidende Kontraindikationen und es kann zu spezifischen Nebenwirkungen kommen. Diese Therapien werden den Ansprüchen der Patienten daher nicht im- 
mer gerecht. Efalizumab (Raptiva) ist als erstes Biologikum in der Schweiz 2004 eingeführt worden und erhielt im Januar 2005 die Kassenzulässigkeit.

Wie stark sich die Psoriasistherapie in den letzten Jahren gewandelt hat, vor allem durch den Einsatz der Biologika, die eine gute Wirksamkeit und Verträglichkeit bei mittelschwerer bis schwerer Psoriasis erzielen, diskutiert Prof. JeanHilaire Saurat, Leiter der Abteilung für Dermatologie und Venerologie am Universitätsspital Genf, als Chairman der Veranstaltung. Ziel des Symposiums "How to manage the new treatment in psoriasis» war es, gemeinsam praktische Erfahrungen im Umgang mit dieser Therapieoption bei Psoriasis auszutauschen.

Prof. Mona Stähle vom Karolinska Institut, Stockholm (Schweden), hebt hervor, dass vor allem Patienten mit einer schweren Psoriasis über einen langen Zeitraum eine erhöhte kardiovaskuläre Morbidität und Mortalität aufweisen [2,3]. Risikofaktoren wie ein abnormales Lipidprofil, Hypertonie, Adipositas und Diabetes sind mit einer schweren Psoriasis eng assoziiert. Ein erhöhtes kardiovaskuläres Risiko dieser Patienten gilt es bei der Wahl der Therapie zu berücksichtigen. Beispielsweise sind TNF- $\alpha$-Inhibitoren bei einer bestehenden Herzinsuffizienz kontraindiziert und Retinoide und Cyclosporine können die Lipidwerte im Blut erhöhen. Psoriasis ist in der Tat nicht nur eine Krankheit der Haut.

\section{Was ist das Besondere des Biologikums Efalizumab?}

Efalizumab, ein monoklonaler, humanisierter Antikörper, moduliert die T-Zellen ohne sie zu zerstören, der Körper bleibt dadurch gegen Infektionen von aussen weiterhin abwehrbereit. Andere Biologika schwächen das Immunsystem. Die Patienten riskieren deutlich mehr Infektionen. Efalizumab blockiert die drei bedeutenden Krankheitsschritte der Plaque-Psoriasis: Auf der Ebene der Lymphknoten hemmt es erstens die Aktivierung naiver T-Zellen durch antigenpräsentierende Zellen. Zweitens wird im Blut die Bindung der T-Zellen an Endothelzellen gehemmt und damit deren Wanderung in die Dermis unterbunden. Drittens hemmt Efalizumab in der Haut die Reaktivierung der Gedächtnis-T-Zellen durch antigenpräsentierende Zellen und verhindert damit das Ausschütten von Zytokinen. Efalizumab ist damit der einzige reversible T-Zell-Modulator, der spezifisch ins Krankheitsgeschehen eingreift. Ausserdem ist Efalizumab einfach in der Anwendung: Die subkutane Injektion wird vom Patienten einmal pro Woche selbst durchgeführt und ist einfach zu erlernen.

\section{Wichtig: Responder von Non- Respondern unterscheiden}

Die Wirksamkeit und Verträglichkeit von Efalizumab wurde in mehreren plazebokontrollierten Phase-III-Studien bei mittelschwerer bis schwerer Psoriasis untersucht. Bisher wurden mehr als 3500 Patienten in klinischen Studien zwischen 1 und 3 Jahren behandelt. Die Wirksamkeitsdaten plazebokontrollierter Studien [4-7] zeigen nach 12-wöchiger Behandlung bei bis zu 39\% der Psoriasispatienten einen PASI 75 (Psoriasis Area and Severity Index: 75\% Reduktion) und bei bis zu 61\% einen PASI 50 (50\% Reduktion). Ausserdem erreichten $30 \%$ der Patienten, die nicht oder nicht mehr auf eine systemische Standardtherapie ansprachen oder bei denen diese kontraindiziert war, nach 12 Wochen Therapie einen PASI 75 [6]. Schon in den ersten 2 Wochen der Behandlung zeigten Responder eine deutliche Wirkung - dies deutet auf einen raschen Wirkeintritt von Efalizumab hin.

Abb. 1. Ansprechrate PASI 75 und PASI 90 während einer Langzeittherapie von 3 Jahren in einer "As-treated-Analyse» [9].

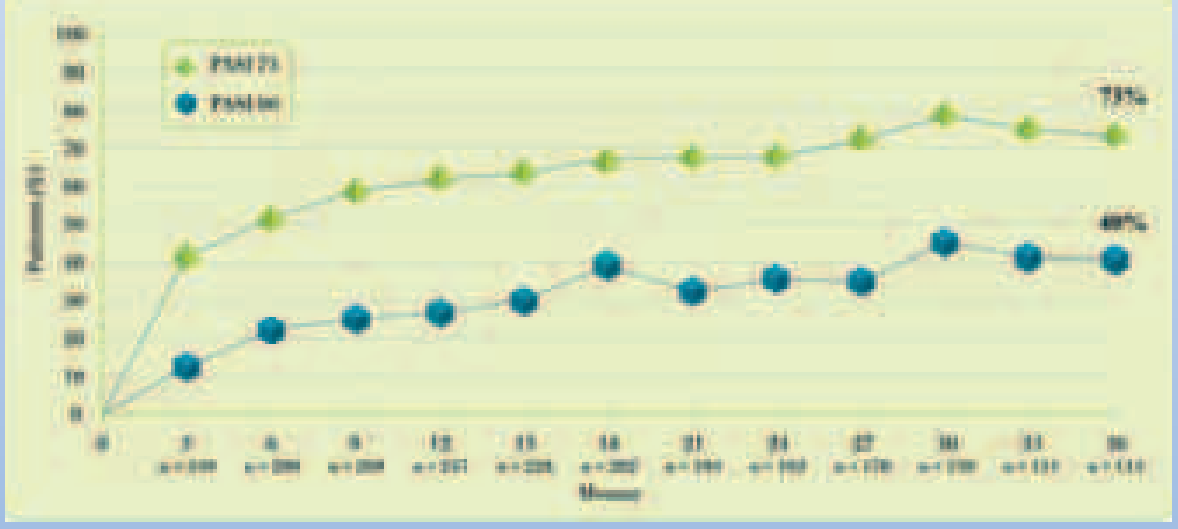

Die Verlängerung der Therapie über die Initialbehandlung hinaus steigert den Prozentsatz der Responder: Nach 24-wöchiger Therapie zeigten bereits 44\% der Patienten einen PASI 75 und $67 \%$ einen PASI 50 [8].

Bei einer Langzeittherapie von 36 Monaten zeigten insgesamt $73 \%$ der Patienten eine Besserung des Hautbildes um 75\% (PASI 75) und 40\% eine Verbesserung um 90\% (PASI 90), in einer «Astreated-Analyse» (Abb. 1).

Wichtig sei zu wissen, so Dr. Selenko-Gebauer, dass Efalizumab gemäss Datenlage keine Wirkung auf eine begleitende Psoriasisarthritis hat. Davon betroffene Patienten benötigten entweder zusätzlich ein NSAR oder alternativ ein Biologikum, das auch gegen die Gelenkbeschwerden wirkt. Ob eine Dauertherapie mit Efalizumab in Frage kommt, entscheide sich in den ersten 12 Wochen. Bessert sich der Hautbefund in dieser Zeit, kann der Erfolg mit der Dauer der Therapie sogar weiter zunehmen. Eine nach 12 Wochen ergebnislose Behandlung sei dagegen ein ausreichender Indikator für ein Nicht-Ansprechen und rechtfertige den Abbruch, so Dr. Selenko-Gebauer. Und: 6-8\% aller mit Efalizumab behandelten Psoriasispatienten seien «inflammatorische NonResponder». Das sind Patienten, bei denen die entzündlichen Hauterscheinungen unter der initialen Therapie stärker statt schwächer werden, erklärt die Dermatologin. Bei einer derart paradoxen Entwicklung sollte das Präparat bereits nach spätestens 8 Wochen abgesetzt werden. 


\section{Auslassversuche gefährden Langzeitansprechen}

Ein Aufflackern der Erkrankung mit der Behandlung von Efalizumab nach primärem Ansprechen sei aber kein Grund, das Mittel umgehend abzusetzen, betont Dr. Selenko-Gebauer. Vielmehr sollten diese Patienten für einige Zeit Zusatztherapien erhalten. Eine Option sei etwa 15-20 mg Methotrexat pro Woche für 4-6 Wochen oder eine forcierte stationäre Lokaltherapie. Dr. Selenko-Gebauer warnt davor, ein gutes Langzeitansprechen mit dem Antikörper durch Versuche zu gefährden, die empfohlene Dosierung (subkutan $1 \mathrm{mg} /$ $\mathrm{kg}$ Körpergewicht/Woche) oder die Applikationsintervalle (wöchentlich) zu strecken. Denn dann sei mit einer schubartigen Verschlechterung des Hautzustandes zu rechnen.

Efalizumab eignet sich für eine dauerhafte Therapie, d.h. der Patient setzt das Medikament nicht mehr ab. Es sollte nicht bei Schwangerschaft angewendet werden. Patienten dürfen während der Behandlung mit Efalizumab keine azellulären Lebendimpfstoffe oder abgeschwächten Lebendimpfstoffe erhalten.

\section{Langfristiges hohes \\ Sicherheits- und \\ Verträglichkeitsprofil}

$\mathrm{Zu}$ den unerwünschten Wirkungen der Behandlung zählen leichte bis mässig akute grippeähnliche Symptome, die aber von den meisten Patienten als «erträglich» eingeschätzt werden und mit Paracetamol einfach behandelt werden können. Sie lassen im Laufe der Behandlungen nach. Bisherige Erfahrungen haben gezeigt, dass es während der Behandlung mit Efalizumab vorwiegend bei den Respondern zu einer Ausbildung von Papeln ähnlich einer Psoriasis guttata kommen kann: Diese treten meist axillär und inguinal etwa 4-8 Wochen nach Beginn der Efalizumab-Therapie auf; sie heilen nach Anwendung topischer Therapien ab, ohne dass die Therapie mit Efalizumab abgebrochen werden muss. Bei einer dauerhaften Anwendung gibt es keine neuen Nebenwirkungen. Im Beobachtungszeitraum von 3 Jahren bei 150 behandelten Patienten konnte kein Tumor-Risiko festgestellt werden. Schwere Infektionen wie bei anderen Biologika traten bei $0,4 \%$ der Patienten auf - eine völlig «realistische» Zahl.

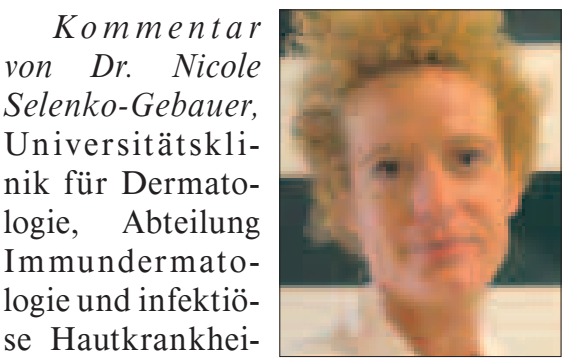
ten, Spezialambu

lanz für Psoriasis und Autoimmundermatosen, Medizinische Universität Wien:

Bei einem Biologikum handelt es sich um ein Eiweiss, das genau in die Schlüsselprozesse der Psoriasis eingreift. Daher ist eine bis dato noch nicht da gewesene Spezifität in der Wirkung gegeben und eine deutlich bessere Verträglichkeit als bei anderen Therapien. Die systemische Psoriasistherapie war bis heute von Medikamenten dominiert, die starke Nebenwirkungen hatten. Keines davon konnte ohne Bedenken kontinuierlich eingenommen werden. Und deshalb ist die Therapie immer eine Pulstherapie gewesen, weil man den Patienten eine dauerhafte Behandlung nicht zumuten konnte. Mit dem Einsatz der Biologika in der Dermatologie ist nunmehr eine gut verträgliche Dauertherapie vorhanden.

Eine Grundvoraussetzung für den Umgang mit Biologika ist das Erkennen von manchmal sehr subtil verlaufenden Nebenwirkungen - das alles gehört grundsätzlich in die Hand des beobachtenden Facharztes. Denn dies ist eine schwierige und verantwortungsvolle Aufgabe.

Laut österreichischem Konsensus sollten Psoriasispatienten mit mittelschwerer bis schwerer Psoriasis mit Biologika behandelt werden - per definitionem Patienten mit einem PASI $>12$ bzw. einer Körperoberflächenbeteiligung $>10 \%$. Meiner Meinung nach sind aber nicht nur diese, sondern z.B. auch Patienten mit palmoplantarer pustulärer Psoriasis als schwere Psoriatiker einzustufen, obwohl sie die genannten Kriterien nicht erfüllen. Die Patienten stehen unter einem enormen Leidensdruck und können zum Teil nicht mehr gehen oder arbeiten. Deshalb sind auch diese
Patienten für mich Kandidaten für Biologika. Fallbeispiele von erfolgreich behandelten Patienten mit palmoplantarer Psoriasis liegen vor [10].

Hinsichtlich der Kostenfrage der Biologika: Es geht sicher nicht darum, in einem leichten Fall, bei dem eine Lokaltherapie ausreicht, Biologika zu verabreichen. Wenn ich jedoch einem Patienten, der 15 Jahre mit PUVA behandelt wurde und der auf herkömmliche Präparate mit schwerster Übelkeit reagiert, durch eine Monotherapie mit einem Biologikum zu einem enormen Zuwachs an Lebensqualität verhelfen kann, so ist der Einsatz der Biologika allemal gerechtfertigt.

Für uns hat Efalizumab einen besonderen Stellenwert, weil das Sicherheitsprofil exzeptionell ist. Das heisst, es gibt bis dato nichts wirklich Vergleichbares. In unserer Klinik sind derzeit etwa 200 Patienten auf Efalizumab eingestellt. Wir haben keine Probleme mit opportunistischen Infektionen, auch keine Neoplasmen, und wir haben ausserdem keinen Wirkungsverlust bei den Patienten. Wir verabreichen Efalizumab auch an Patienten mit einer latenten Tuberkulose und verwenden es auch bei Psoriatikern mit Herzerkrankungen und Hepatitis. Prinzipiell wenden wir es als Biologikum der Wahl an. Der typische Patient für das Efalizumab ist derjenige mit einer wirklich mittelschweren bis schweren Psoriasis, der keine Gelenksprobleme hat.

\author{
Berichterstattung: \\ Dr. Ellen Heitlinger \\ $\mathrm{H}+\mathrm{O}$ communications ag, Baar \\ Mit Unterstützung von \\ Serono Pharma Schweiz \\ Steinhauserstrasse 74, Postfach \\ CH-6301 Zug \\ info@psoriasisweb.ch \\ www.raptiva.ch
}

\section{Literatur}

1 Nickoloff BJ, et al: Recent insights into the immunopathogenesis of psoriasis provide new therapeutic opportunities. J Clin Invest 2004;113:1664-1675.

2 Malbris L, et al: Psoriasis is associated with lipid abnormalities at the onset of skin disease. J Am Acad Dermatol 2006; 54:614-621. 
3 Henseler T, et al: Disease concomitance in psoriasis. J Am Acad Dermatol 1995;32: 982-986.

4 Lebwohl MG, et al: A novel targeted T-cell modulator, efalizumab, for plaque psoriasis. N Engl J Med 2003;349:2004-2013.

5 Gordon KB, et al: Efalizumab for patients with moderate to severe plaque psoriasis: a randomized, controlled trial. JAMA 2003;290:3073-3080.

6 Sterry W, et al: Clinical Experience Acquired with Raptiva (CLEAR) trial in patients with moderate-to-severe plaque psoriasis: results from extended treatment in an international, phase III, placebo-controlled trial. JDDG, im Druck.

7 Leonardi CL, et al: Extended efalizumab therapy improves chronic plaque psoriasis: results from a randomized phase III trial. J Am Acad Dermatol 2005;52:425433.

8 Menter A, et al: Efficacy and safety observed during 24 weeks of efalizumab therapy in patients with moderate to severe plaque psoriasis. Arch Dermatol 2005; 141:31-38.

9 Poulin Y, et al: A favourable benefit/risk ratio with efalizumab: a review of the clinical evidence. J Cutan Med Surg 2005; 1: 10-17.

10 Fretzin SY, et al: Efficacy and safety of efalizumab for patients with palmoplantar pustulosis (Poster). Meet Am Acad Dermatol, New Orleans, Februar 2005.

\section{Pédiatril pour la peau délicate des bébés}

\section{La peau délicate des bébés}

Les structures cutanées du bébé et de l'adulte sont proches, mais présentent des particularités dont il faut tenir compte.

- L'épiderme du bébé est comparable à celui de l'adulte, mais l'hydratation de la couche cornée est moindre ce qui donne une impression de peau sèche et légèrement râpeuse parfois.

- Le derme, quant à lui, est plus riche en fibroblastes, mais moins dense en collagène et fibres élastiques. Les glandes sébacées produisent un sébum en grande quantité dans les premières semaines de vie (ce qui explique l'apparition possible d'acné), mais cette sécrétion, due principalement aux hormones résiduelles de la maman, diminue au cours des premiers mois et ne permet pas de réaliser un film hydrolipidique aussi protecteur que celui de l'adulte.

- L'absorption percutanée des soins appliqués sur la peau est comparable à celle observée chez l'adulte, mais le rap- port surface/poids est bien supérieur pour un bébé, ce qui multiplie le risque d'intoxication percutanée. Ce risque d'intoxication percutanée est d'autant plus important que la surface corporelle du bébé est facilement couverte en totalité par un produit topique.

Les particularités de la peau du bébé justifient la grande rigueur avec laquelle les Laboratoires dermatologiques Avène ont formulé Pédiatril. La charte de formulation et les études cliniques réalisées en conditions d'usage sont garants de la qualité de ces soins.

\section{La charte de formulation Pédiatril}

La gamme Pédiatril se caractérise par une charte spécifique, qui est rappelée sur les conditionnements des produits de soin.

Tous les produits Pédiatril sont:

- riches en Eau thermale d'Avène,

- formulés avec un minimum d'ingrédients sélectionnés pour leur grande douceur et leur parfaite tolérance,

- sans parfum, sans colorant, sans alcool et sans conservateur paraben,

- testés par des pédiatres et des dermatologues, 
- adaptés aux peaux les plus fragiles même à tendance atopique.

Tous ces produits ont bénéficié d'évaluations rigoureuses et présentent une très haute tolérance.

\section{La gamme Pédiatril - Tout le savoir-faire Avène au service de la peau délicate des bébés}

Pour l'hygiène quotidienne:

Pédiatril Mousse lavante corps et cheveux

Elle élimine en douceur les impuretés de la peau et du cuir chevelu en évitant tout dessèchement cutané. Sa mousse légère et onctueuse ne pique pas les yeux.

\section{Pour le soin de bébé:}

\section{Pédiatril Crème de soin}

La Crème de soin Pédiatril apaise, hydrate et nourrit la peau délicate du nourrisson et du bébé. Les agents hydratants et nutritifs, harmonieusement équilibrés, protègent en douceur la peau délicate du nourrisson et du bébé. Onctueuse et non grasse, la Crème de soin Pédiatril pénètre facilement et laisse la peau douce et veloutée à souhait.

\section{Pédiatril Gel «croûtes de lait»}

Le Gel «croûtes de lait» Pédiatril est un soin humectant et émollient qui élimine les squames épaisses et grasses du cuir chevelu du nourrisson (appelées croûtes de lait). Parmi ces actifs, le lactamide, actif naturel biologique composant du «natural moisturizing factor», a un effet humectant et kératorégulateur doux. Grâce à l'association glycérine, panthénol et huile d'amande douce, les croûtes de lait se détachent et s'éliminent facilement. La base gélifiée aqueuse du gel présente l'avantage de se rincer facilement sans laisser de résidu gras.

\section{Pédiatril Pâte à l'eau}

La Pâte à l'eau Pédiatril prévient les rougeurs et irritations du siège du nourrisson et du bébé en isolant la peau des urines et des selles.

L'oxyde de zinc, avec ses propriétés antibactériennes et protectrices, favorise la régénération des zones irritées. Il prévient également le développement des bactéries, mettant ainsi la peau à l'abri de l'agression des urines et des selles. L'huile d'amande douce calme les sensations d'inconfort et adoucit la peau. La glycérine enfin apporte son action hydratante. Efficace, la Pâte à l'eau Pédiatril se distingue aussi par ses qualités cosmétiques: elle s'applique aisément sans coller, sans laisser de traces et s'élimine très facilement.

\section{Pediatril für zarte Babyhaut}

\section{Die zarte Haut von Babys}

Die Hautstrukturen von Babys und Erwachsenen sind ähnlich, aber sie weisen Besonderheiten auf, die man berücksichtigen muss.

- Die Epidermis von Babys ist vergleichbar mit derjenigen von Erwachsenen, aber der Feuchtigkeitsgehalt der Hornschicht ist niedriger, was die Haut trockener und rauer erscheinen lässt.

- In der Dermis sind mehr Fibroblasten, aber weniger Kollagen- und Elastinfasern. Die Talgdrüsen produzieren viel Talg in den ersten Wochen des Lebens (das erklärt das mögliche Auftreten von Akne), aber dieser Überschuss, hauptsächlich ausgelöst durch die Hormone der Mutter, vermindert sich im Laufe der ersten Monate und verhindert den Aufbau eines Hydrolipidfilms, der genau so schützend ist wie der des Erwachsenen.

- Die Hautabsorption der angewendeten Pflege ist vergleichbar mit derjenigen, die beim Erwachsenen beobachtet wird, aber das Verhältnis Fläche/Gewicht ist bei einem Baby viel grösser, was das Risiko einer perkutanen Vergiftung multipliziert. Dieses Risiko der perkutanen Vergiftung ist umso höher, als die Körperoberfläche des Babys schnell durch ein topisches Produkt komplett bedeckt werden kann.

Die Besonderheiten der Babyhaut begründen die grosse Sorgfalt, mit welcher die Laboratoires dermatologiques Avène Pediatril formuliert haben. Die Zusammensetzung und die klinischen Forschungen, die unter Gebrauchsbedingungen durchgeführt wurden, sind die Garanten für die Qualität der Pflegeprodukte.

\section{Die Zusammensetzung von Pediatril}

Die Linie Pediatril ist durch eine spezifische Zusammensetzung charakterisiert, die sich in der Aufmachung der Pflegeprodukte widerspiegelt.

Alle Produkte der Pediatril-Babypflege sind:

- reich an Avène-Thermalwasser,

- formuliert mit einer minimalen Menge an Inhaltsstoffen, die aufgrund ihrer Milde und hohen Hautverträglichkeit ausgewählt wurden,

- ohne Duftstoffe, ohne Farbstoffe, ohne Alkohol und ohne herkömmliche Konservierungsstoffe,

- von Kinderärzten und Dermatologen getestet,

- für empfindlichste, auch atopische Hauttypen geeignet.

Alle Produkte wurden unter strengsten Produktionsnormen auf ihre hohe Hautverträglichkeit getestet.

\section{Die Linie Pediatril - Das ganze Know-how von Avène im Dienste der zarten Haut der Babys}

Für die tägliche Hygiene:

Pediatril Baby-Reinigungsschaum

\section{für Haut und Haare}

Der Schaum entfernt sanft die Unreinheiten der Haut und des Haarbodens ohne die Haut dabei auszutrocknen. Der leichte und cremige Schaum brennt nicht in den Augen.

Für die Babypflege:

Pediatril Pflegecreme

Die Pflegecreme Pediatril beruhigt, spendet Feuchtigkeit und nährt die feine Haut von Säuglingen und Babys. Feuchtigkeitsspendende und nährende Wirkstoffe bringen die Haut des Babys ins Gleichgewicht und schützen sie sanft. Cremig und nicht fettend, zieht die Pflegecreme Pediatril leicht ein und lässt die Haut samtweich werden.

\section{Pediatril Milchschorf-Gel}

Das Milchschorf-Gel Pediatril ist ein Feuchtigkeitsspender, der die dichten und fettigen Schuppen des Haarbodens von Säuglingen entfernt (so genannter Milchschorf). Unter diesen Wirkstoffen hat das Lactamid, ein na- 
turbiologischer Wirkstoff, der im «natural moisturizing factor» enthalten ist, einen feuchtigkeitsspendenden und sanft keratolytischen Effekt. Dank der Kombination von Glycerin, Panthenol und Süssmandelöl lässt sich der Milchschorf leicht entfernen. Die wässrige Gel-Konsistenz hat den Vorteil, dass beim Abwaschen kein fettiger Rest zurückbleibt.

\section{Pediatril Baby-Wundpflegecreme}

Die Wundpflegecreme Pediatril beugt Rötungen und Hautreizungen von Säuglingen und Babys vor, indem die Haut vom Urin und vom Stuhl isoliert wird. Das Zinkoxyd mit seinen hauterneuernden Eigenschaften beschleunigt die Regeneration der Haut an den angegriffenen Stellen. Es beugt der Bildung von Bakterien vor und schützt die Haut vor Reizungen durch Urin und Stuhl. Das Mandelöl mindert das unangenehme Hautgefühl und macht die Haut weich. Das Glycerin versorgt schliesslich die Haut mit Feuchtigkeit. Die Baby-Wundpflegecreme zeichnet sich auch durch ihre kosmetischen Qualitäten aus: sie lässt sich ohne weiteres auftragen ohne zu kleben, ohne Spuren zu hinterlassen und sie lässt sich leicht entfernen.

\section{La nouvelle} gamme Homme à I'eau thermale d'Avène:

Des formules dédiées aux peaux sensibles!

Barbe dure, barbe normale ou peau à problèmes, les nouveaux soins de rasage et d'après-rasage des Laboratoires dermatologiques Avène offrent enfin une réponse spécifique adaptée à la peau de l'homme.

Plus actuelle, cette nouvelle gamme se distingue aussi par ses formules complètes qui font du rasage un moment de soin. Ses actifs, complémentaires et synergiques, ont 3 objectifs:

- apaiser, grâce à l'Eau thermale d'Avène, naturellement apaisante et anti-irritante, et grâce à l' $\alpha$-bisabolol;

- assainir, grâce au triclosan, antibactérien de référence qui prévient tout risque bactérien survenant lors de microcoupures ou en cas de lame souillée;

- hydrater, grâce à la glycérine, qui apporte souplesse et confort à la peau.

Tout comme les soins de rasage, les soins après-rasage apaisent, assainissent et hydratent, mais un actif complémentaire vient renforcer l'efficacité: le sucralfate.
Cet actif réparateur, parfaitement adapté aux problèmes de microcoupures, protège les lésions liées au rasage.

Favorisant la réparation des vaisseaux, le sucralfate inhibe également les médiateurs de l'irritation et stimule la prolifération des kératinocytes.

La nouvelle gamme Homme Avène, ce sont donc de nouvelles formules, de nouveaux produits pour offrir aux hommes des soins adaptés à leur peau sensible.

Pour le rasage:

- Gel de rasage, pour tous les types de barbes et plus spécifiquement les peaux à imperfections

- Mousse à raser, pour les barbes normales

- Crème à raser, pour les barbes dures

Soins après-rasage:

- Fluide après-rasage, pour les peaux normales à mixtes et contenant du Cucurbita pepo séborégulateur

- Baume après-rasage, pour les peaux sèches

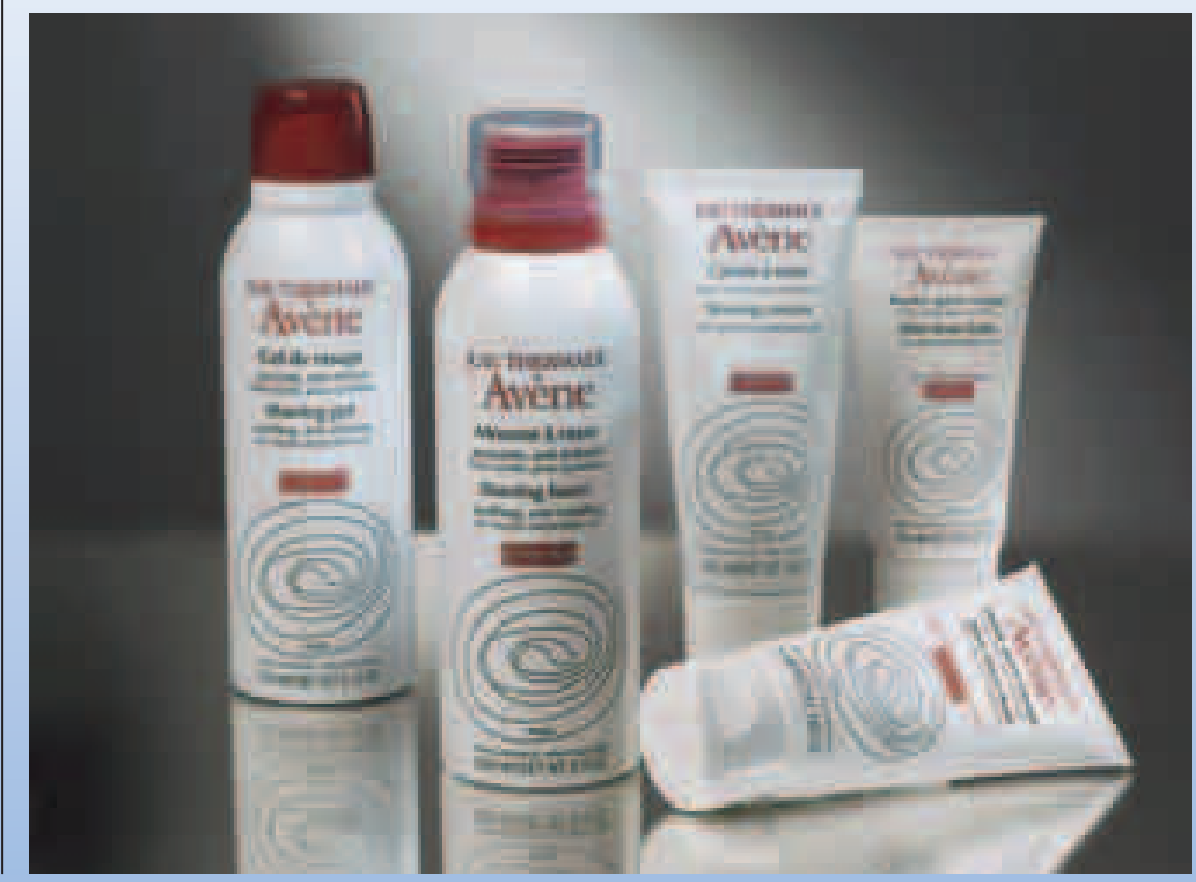




\section{Die neue Pflege-} linie für den Mann mit Avène-

\section{Thermalwasser:}

Zusammensetzungen, die der empfindlichen Haut angepasst sind!

Harter oder normaler Bart, Problemhaut - die neue Avène Rasier- und After-Shave-Pflegelinie der Laboratoires dermatologiques Avène bietet nun eine spezifische, an die Männerhaut angepasste Antwort.

Sie ist topaktuell und unterscheidet sich auch durch ihre kompletten Formulierungen, die die Rasur zur Pflege machen. Die ergänzenden und synergetischen Wirkstoffe haben 3 Ziele:

- beruhigen, dank dem Avène-Thermalwasser, natürlich beruhigend und reizlindernd, und dank dem $\alpha$-Bisabolol;
- desinfizieren, dank dem Triclosan, einem bakteriostatischen Wirkstoff, der allen bakteriellen Risiken (bei kleinen Hautschnitten oder verschmutzter Rasierklinge) vorbeugt;

- Feuchtigkeit spenden, dank dem Glyzerin, das der Haut Geschmeidigkeit und Wohlgefühl verleiht, ohne einen Fettfilm zu hinterlassen.

Wie auch die Rasier-Pflegeprodukte beruhigt und desinfiziert der AfterShave-Balsam und spendet Feuchtigkeit, zudem verstärkt ein ergänzender Wirkstoff die Wirksamkeit: Sucralfat.

Dieser regenerierende Aktivstoff ist perfekt auf die Problematik kleiner Hautverletzungen bei der Rasur abgestimmt und schützt die Verletzungen durch die Rasierklinge.

Das Sucralfat begünstigt die Regenerierung der Gefässe, lindert ebenfalls Reizungen und stimuliert die Vermehrung von Keratinozyten.
Die neue Pflegelinie für den Mann zeichnet sich durch neue Formulierungen und neue Produkte aus, die speziell an die empfindliche Männerhaut angepasst sind.

Für die Rasur:

- Rasiergel, für alle Barttypen und vor allem für Haut mit Unreinheiten

- Rasierschaum, für den normalen Bart

- Rasiercreme, für den harten Bart

Nach der Rasur:

- After-Shave-Fluid, für normale und Mischhaut, enthält

Kürbiskernextrakt

- After-Shave-Balsam, für trockene Haut

\author{
Pierre Fabre Suisse (SA) \\ Gewerbestrasse 25 \\ CH-4123 Allschwil \\ Tel. 0614878900 \\ Fax 0614878901 \\ E-Mal caroline.welsch@pierre-fabre.com
}

\section{Deux déma- quillants pour les yeux de Louis Widmer destinés aux femmes qui portent des lentilles de contact}

Louis Widmer a mis au point deux démaquillants pour les yeux ne contenant ni parfum ni colorant, tout particulièrement pour les personnes qui ont les yeux sensibles et les femmes qui portent des lentilles de contact. Les deux produits ont donné des résultats optimaux dans les tests cliniques.

Le Démaquillant yeux - lotion élimine doucement et parfaitement le maquillage hydrosoluble. Le D-panthénol améliore le pouvoir hydratant, protège contre les irritations et calme la peau.

Le Démaquillant yeux - waterproof - non-oily est un produit en 2 phases qui convient particulièrement pour le maquillage waterproof. Des huiles végéta-

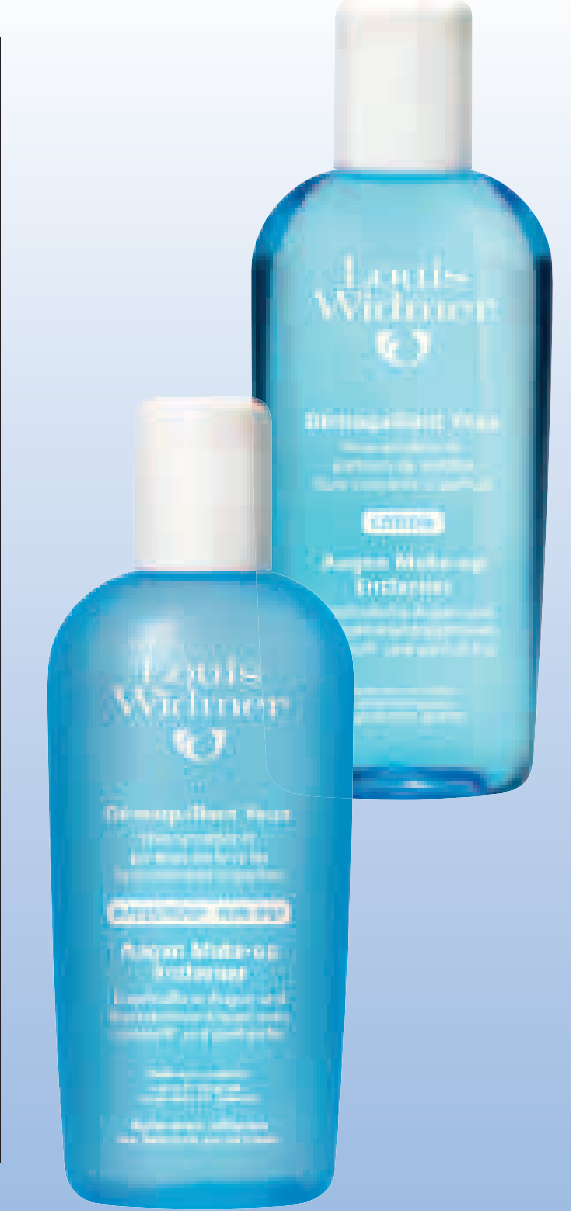

les (squalanes) protègent la peau, le Dpanthénol améliore le pouvoir hydratant et calme.

\section{Test de tolérance ${ }^{1}$}

Un institut allemand spécialisé en dermatologie a soumis les deux démaquillants pour les yeux à un test de tolérance: chacun des deux produits a été appliqué chez 50 sujets, par patch-test sur le dos, le but étant d'évaluer leur tolérance. Aucun des sujets n'a présenté d'irritation ou de réaction allergique. La tolérance des deux démaquillants pour les yeux a été jugée excellente.

\section{Test ophtalmologique ${ }^{2}$}

25 femmes ont utilisé le Démaquillant yeux - Lotion et 25 autres femmes le Démaquillant yeux - Waterproof - non-oily pendant 4 semaines. Lors de l'examen ophtalmologique final, l'oph-

1 Test de tolérance cutanée, expertise effectuée par un institut allemand spécialisé en dermatologie.

2 Test ophtalmologique, effectué par Dr Marco Krugmann, ophtalmologue. 
talmologiste, le Dr Marco Krugmann, n'a constaté des irritations des paupières, de la cornée ou des yeux chez aucune des femmes. Du point de vue ophtalmologique, les deux produits peuvent être considérés comme remarquables.

Les deux démaquillants pour les yeux se caractérisent par une très bonne acceptabilité et une sensation cutanée agréable. Ils sont très économiques à l'utilisation, ils soignent et protègent la peau.

Louis Widmer SA

Rietbachstrasse 5

CH-8952 Schlieren

Tél. +41434337700

Fax +41434337799

E-mail info@louis-widmer.com

\section{Zwei Augen Make- up Entferner von Louis Widmer für Kontaktlinsen- trägerinnen}

Speziell für Personen mit empfindlichen Augen sowie für Kontaktlinsenträgerinnen hat Louis Widmer zwei Make-up Entferner ohne Parfüm- und Farbstoffzusätze entwickelt. Beide Produkte haben optimale Ergebnisse in klinischen Tests erzielt.

Der Augen Make-up Entferner - Lotion entfernt wasserlösliches Make-up schonend und gründlich. D-Panthenol verbessert das Feuchthaltevermögen, schützt vor Irritationen und beruhigt die Haut.

Der Augen Make-up Entferner - Waterproof-non-oily ist ein 2-Phasen-Produkt und eignet sich speziell für wasserfestes Make-up. Pflanzliche Öle (Squalane) schützen die Haut, D-Panthenol verbessert das Feuchthaltevermögen und beruhigt.

\section{Verträglichkeitstest ${ }^{1}$}

Ein spezialisiertes dermatologisches Institut in Deutschland unterzog die beiden Augen Make-up Entferner§ einem Verträglichkeitstest. Dabei wurden die beiden Produkte an je 50 Probanden mittels Patch-Test am Rücken auf ihre Verträglichkeit getestet. Bei keinem der Probanden zeigten sich Irritationen oder allergische Reaktionen. Die Verträglichkeit der beiden AugenMake-up-Entferner wurde als hervorragend bezeichnet.

\section{Augenärztlicher Test ${ }^{2}$}

Beide Augen Make-up Entferner wurden während 4 Wochen von je 25 Probandinnen verwendet. Der Facharzt für Augenheilkunde Dr. Marco Krugmann stellte bei der ophthalmologi-

${ }^{1}$ Verträglichkeitstest durchgeführt von einem spezialisierten dermatologischen Institut in Deutschland.

2 Augenärztlicher Test durchgeführt von Dr. Marco Krugmann, Facharzt für Augenheilkunde. schen Abschlussuntersuchung bei keiner der Probandinnen Reizungen der Lider, der Hornhaut sowie der Augen fest. Aus augenärztlicher Sicht können beide Produkte als hervorragend bezeichnet werden.

Beide Augen-Make-up-Entferner zeichnen sich durch eine hohe Akzeptanz sowie ein angenehmes Hautgefühl aus. Sie sind äusserst sparsam im Verbrauch und haben eine pflegende und hautschonende Wirkung.

Louis Widmer AG

Rietbachstrasse 5

CH-8952 Schlieren

Tel. +41434337700

Fax +41434337799

E-Mailinfo@louis-widmer.com 